\title{
La cellule R1 des microfilaires (Nematoda), initiale du mésenchyme
}

\author{
par Odile BAIN \\ Laboratoire de Zoologie (Vers) associé au C.N.R.S., Muséum National d'Histoire Naturelle \\ ( $P^{r}$ A.-G. Chabaud), 57, rue Cuvier, F. 75 - Paris $\left(5^{\circ}\right)$ \\ et Centre Muraz de Bobo-Dioulasso (Haute-Volta)
}

\begin{abstract}
Résumé
L'étude des premières heures du développement des microfilaires (essentiellement Setaria labiatopapillosa) chez l'hôte intermédiaire permet de préciser la fonction de certaines cellules particulières et l'origine de quelques organes: les cellules R2 à R4 servent à l'élaboration du rectum; la cellule R1 est à l'origine de la musculature de la Filaire adulte; l'intestin se forme à partir de 5 initiales visibles dès le début du développement; l'œsophage se construit autour d'un axe cuticulaire qui s'étend de l'extrémité apicale jusqu'au voisinage du noyau intestinal le plus antérieur.
\end{abstract}

\section{Summary}

The study of the first hours of development of Microfilaria (exclusively Setaria labiatopapillosa) in the intermediary host has unable the author to assess the fate of some peculiar cells and the origin of some organs. The R2 to R4 cells are involved in the production of the rectum. The $\mathrm{R} 1$ cell will give rise to the adult Filaria musculature. The intestine is formed by five initial cells which become apparent from the early stages of development. The oesophagus is fashioned round a cuticular axis extending from the apical end to the proximity of the most anterior intestinal nucleus. 


\section{Introduction.}

Feng, en 1936, montre que, chez la microfilaire, les 4 «G-cells » de Rodenwaldt (1908) n'interviennent pas dans la formation de l'ébauche génitale mais forment le rectum; il les nomme « R-cells». Ses idées sont admises avec quelques variantes par Kobayashi (1940) mais non par tous les auteurs; pour R. Iyengar (1956), la R1 est une cellule génitale mais les autres cellules forment l'intestin (R2 et R3) et le rectum (R4) ; pour Taylor (1960), la R1 s'incorpore à R2 et R3 pour former l'intestin ; pour Schacher (1962) et Bain (1969 $a$ et $b$ ), les cellules R2, R3 et R4 forment le rectum; le devenir de $\mathrm{R} 1$, par contre, reste imprécis mais les divisions répétées de cette cellule laissent soupçonner son rôle dans la formation de l'intestin.

L'étude du développement d'une microfilaire particulièrement grande, Setaria labiatopapillosa (Alessandrini, 1838), nous a permis de suivre les descendants de la cellule R1 et de montrer qu'elle n'entre pas dans la formation de l'appareil digestif, mais qu'elle est à l'origine du fourreau musculaire de la Filaire adulte.

\section{Matériel.}

Il est principalement originaire de Haute-Volta. S. labiatopapillosa est fourni par un veau infesté expérimentalement à Bobodioulasso avec la souche de Tingrela; le vecteur, Aedes aegypti, est une souche de Kongolikan entretenue à la Section Entomologique du Centre Muraz.

Deux autres Filaires sont aussi étudiées: Wuchereria bancrofti (Cobbold, 1877) (souche de Tonogosso) chez Anopheles gambiae A (souche de Koupela) et Foleyella candezei (Fraipont, 1882) (souche de Pala) chez A. aegypti (souche de Kongolikan).

La température varie de $25^{\circ}$ à $30^{\circ} \mathrm{C}$.

Les observations sont effectuées à frais, après coloration vitale pour les microfilaires et sans coloration pour les larves en développement.

Certains détails de la structure de la microfilaire sont étudiés, en microscopie électronique, chez Foleyella furcata (Linstow, 1899) provenant d'un Caméléon malgache.

\section{Formation du rectum et de l'intestin.}

1) Observations chez Setaria labiatopapillosa.

Chez les microfilaires, seules les cellules sous-cuticulaires, excrétrices et rectales sont visibles; le corps interne est allongé, régulièrement ponctué ou formé de gros globules réfringents (cீ. Bain, sous presse).

- $23 \mathrm{~h}$ après le repas infectant (fig. $1 \mathrm{~A}$ ), les 4 noyaux rectaux grossissent et migrent vers le pore anal, les noyaux R2 à R4, à l'exclusion du noyau R1, sont enve-

(1) En plus des cellules $R$ et des cellules intestinales, on observe chez la jeune larve quatre petites cellules groupées en deux paires latéro-ventrales, à mi-hauteur de l'intestin. En raison de leur emplacement et de leur nombre nous pensons que ce sont les initiales génitales (nommées « $G$ » sur nos figures) (selon Nigon, in Grassé, l'ébauche génitale des Nématodes est formée par quatre cellules) 


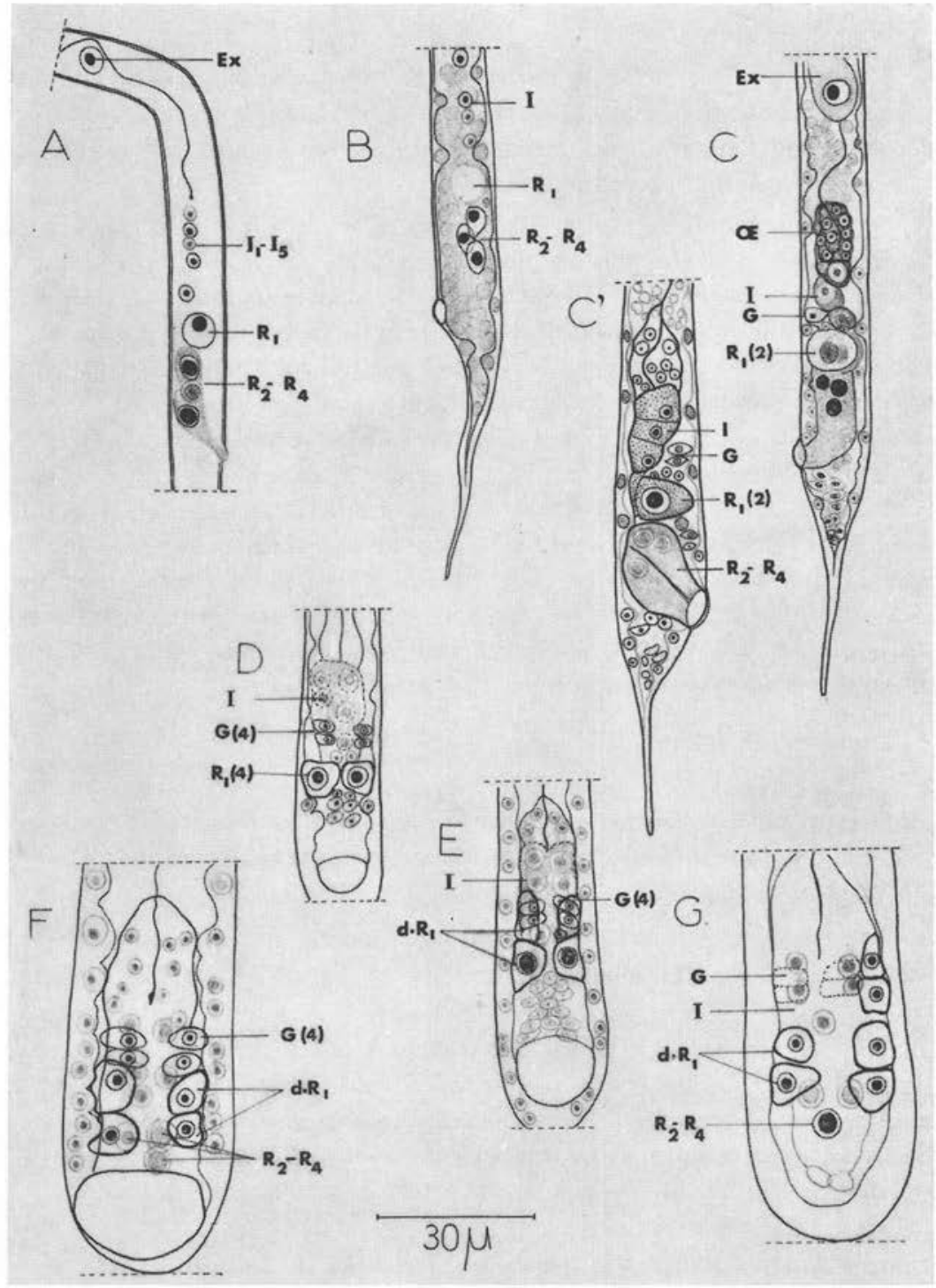

Fig. 1. - S. labiatopapillosa. - A : $23^{e} \mathrm{~h}$; les cellules R2 à R4 sont regroupées et entourées de cytoplasme; le noyau R1 est gonflé ; les 5 initiales intestinales et l'axe œesophagien sont visibles. $\mathrm{B}$ et $\mathrm{C}: 30^{\mathrm{e}} \mathrm{h}$; en $\mathrm{B}, \mathrm{R} 1$ juste avant la $1^{\text {re }}$ division; en $\mathrm{C}, \mathrm{R} 1$ a donné 2 cellules filles. $-\mathrm{C}^{\prime}$ : $40^{\circ} \mathrm{h}$; le rectum est formé par les cellules R2 à R4; l'intestin est relié au rectum par un isthme cytoplasmique ; le bourgeon œsophagien postérieur est formé. - D et E: $50^{\mathrm{e}} \mathrm{h}$, au stade des 4 celiules filles de R1; en vue ventrale (D), on voit en trait épais 2 cellules R1 latéro-ventrales; audessus, en trait plus mince, les 4 cellules $\mathrm{G}$; en pointillé, l'intestin formé par 5 cellules; en vue dorsale (E), une autre larve montre que la descendance de R1 est formée de 8 cellules (4 sont représentées). - F et $\mathrm{G}: 72^{\circ} \mathrm{h}$, respectivement en vue ventrale et dorsale ; en $\mathrm{G}$, sur le côté droit, les 2 descendants de R1 forment une file de 4 cellules (éch. $30 \mu$ )

SYMBOLE DES FIGURES : C.E. : corde ectodermique. d. R1 : descendance de R1. Ex. : cellule excrétrice. G. : 4 cellules ventrales qui pourraient constituer l'ébauche génitale. I. : les 5 initiales intestinales. Oe : œsophage. pr.: 2 cellules formant la jonction intestin-rectum (prérectum). R1: cellule R1, initiale mésenchymateuse. R1 (2): $1^{\text {re }}$ division de R1; R1 (4): $2^{\text {e }}$ division de R1. R2-R4: les 3 cellules rectales 
loppés par le même cytoplasme qui est relié au pore anal ; il existe 2 noyaux latéroventraux (R2 et R3) et un noyau dorsal R4; un axe cuticulaire œsophagien apparaît entre l'apex et le début du corps interne.

Au cours des heures suivantes, les noyaux R2 à R4 ne se divisent pas; leur cytoplasme s'individualise de plus en plus nettement et, dès la $30^{\circ} \mathrm{h}$, l'ébauche rectale est facilement identifiable (fig. 1 C). Par la suite, le rectum augmente de taille; 2 petits noyaux, l'un ventral et l'autre dorsal, s'incorporent à la région proximale du rectum (fig. 2) ; leur origine n'est pas élucidée. Au $1^{\text {er }}$ stade, l'épithélium péri-anal s'invagine et contribue à la formation du rectum (segment distal).

- Chez la larve de $23 \mathrm{~h}$, entre l'axe cuticulaire œophagien et la R1, 5 petits noyaux ronds et vésiculeux apparaissent étagés à l'ancien emplacement du corps interne ( 2 sur le flanc gauche et 3 sur le flanc droit). Ils grossissent, s'entourent de cytoplasme sans se diviser, se tassent les uns contre les autres et forment une ébauche intestinale de 5 cellules; en arrière, elle est reliée au rectum par un pont cytoplasmique étroit, en avant elle est en contact avec un épais bourgeon œsophagien. L'intestin s'accroît sans multiplication cellulaire jusqu'à la fin du $3^{\circ}$ jour (fig. $1 \mathrm{~F}$ ) puis ces cellules commencent à se diviser (fig. $\left.2 \mathrm{~A}, 92^{\circ} \mathrm{h}\right)(1)$.

2) Autres espèces observées.

- Wuchereria bancrofti, les phénomènes sont identiques; le rectum de 3 cellules (R2 à R4) se soude à l'ébauche intestinale de 5 cellules par un étroit pont cytoplasmique ; 2 petits noyaux marquent la jonction de ces 2 organes (fig. $2 \mathrm{C}, \mathrm{D}, \mathrm{E}, 67^{\circ} \mathrm{h}$, fig. F, 4 jours et demi).

- Foleyella candezei, la figure $2 \mathrm{G}$ ( $5^{\mathrm{e}}$ jour) montre que cette Filaire a la même organisation que les espèces précédentes.

Les observations effectuées sur ces 3 espèces de Filaires, appartenant à des familles ou sous-familles différentes (Setariidae, Onchocercidae: Onchocercinae et Dirofilariinae), confirment que le rectum est constitué par les cellules R2 à R4, auxquelles s'ajoutent 2 petites cellules apicales dont l'origine n'est pas élucidée; elles montrent aussi que l'intestin se forme indépendamment des cellules rectales à partir de 5 initiales; ce chiffre est constant quelque soit la famille étudiée.

\section{Formation des champs musculaires; les divisions de la cellule Rl.}

A) Interprétation des cellules sous-cuticulaires de la microfilaire.

Dans un article précédent (Bain, $1969 a$ ), nous avons dit que les cellules musculaires des microfilaires étaient très probablement des cellules ectodermiques, capables de secréter de la cuticule, et dont le cytoplasme s'est secondairement différencié en myofibrilles.

(1) En plus des cellules $R$ et des cellules intestinales, on observe chez la jeune larve quatre petites cellules grouupées en deux paires latéro-ventrales, à mi-hauteur de l'intestin. En raison de leur emplacement et de leur nombre, nous pensons que se sont les initiales génitales (nommées « $G$ » sur nos figures) (selon Nigon, in Grassé, l'ébauche génitale des Nématodes est formée par quatre cellules). 

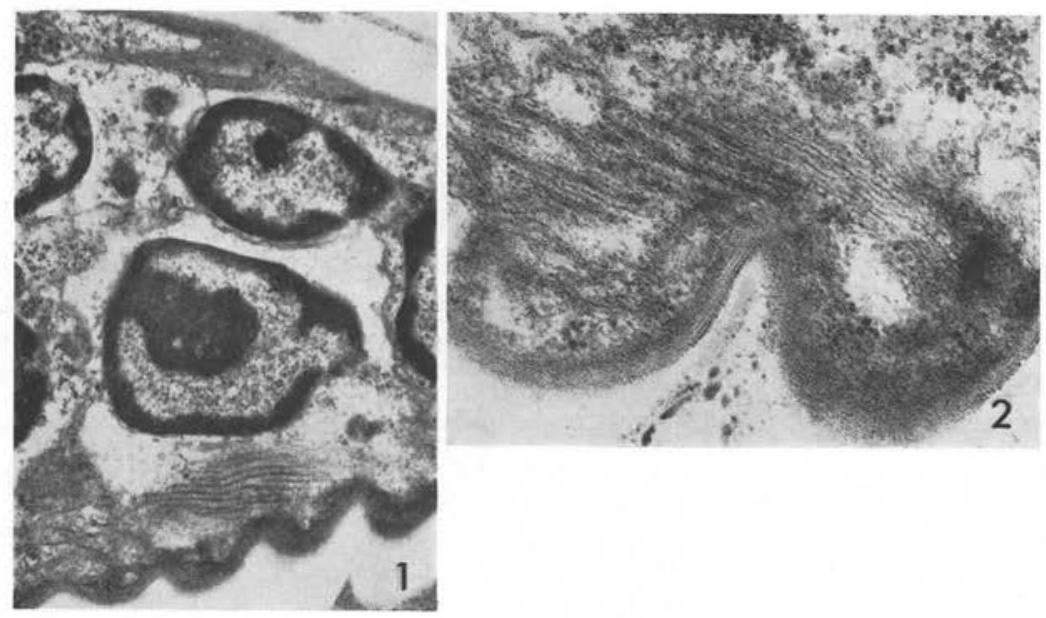

Рното 1. - Coupe d'une microfilaire de $F$. furcata montrant une cellule souscuticulaire avec la base myofibrillaire et le cytoplasme apical renfermant le noyau $(\mathrm{G}=10.000)$

Рното 2. - F. furcata; détail d'une cellule sous-cuticulaire montrant qu'elle est directement en contact avec la cuticule $(G=23.900)$

Des observations de la microfilaire, $F$. furcata, au microscope électronique (2) paraissent confirmer cette opinion : chaque cellule musculaire est constituée d'une base myofibrillaire contractile et d'un apex au cytoplasme indifférencié, contenant le noyau (photo 1); la base de ces cellules est directement en contact avec la cuticule (photo 2).

B) Les divisions de la cellule R1.

1) Observations chez S. labiatopapillosa.

Chez la microfilaire, le noyau R1 est proche du groupe R2 à R4; au début du dévelopement, il suit le mouvement de migration des autres cellules rectales vers le pore anal. Mais vers la $23^{\mathrm{e}} \mathrm{h}$, son comportement devient particulier : il devient très volumineux et se divise une première fois (fig. $1 \mathrm{~A}, \mathrm{~B}$ et $\mathrm{C}$ ) pour donner 2 cellules filles latéro-ventrales (fig. 1 C'); ces cellules ont un cytoplasme bien délimité et sont situées au-dessus du rectum, contre le pont cytoplasmique qui relie cet organe à l'intestin. Les 2 cellules filles se divisent à leur tour pour donner 2 nouvelles cellules latéro-dorsales. Ces 4 cellules issues de R1 sont volumineuses et se reconnaissent aisément; elles sont situées dans le même plan transversal, vers la périphérie de la larve ; elles entourent la portion distale de l'intestin mais n'entrent pas en contact avec lui (fig. $1 \mathrm{D}, 40^{\circ} \mathrm{h}$ ).

(2) Le matériel est fixé au Glutharaldéhyde au pH 7,4, postfixé à l'acide osmique ; l'amélioration des contrastes est effectuée à l'acétate d'uranyle et au citrate de plomb. 
Plus tard, 1 cellule de grande taille apparaît juste en avant de chaque cellule fille de R1 (fig. $1 \mathrm{E}, 50^{\circ} \mathrm{h}$ ); au cours des heures suivantes, de nouvelles cellules s'alignent sur les précédentes (fig. $1 \mathrm{~F}$ et $\mathrm{G}, 72^{\circ} \mathrm{h}$ ) ; l'ensemble de ces cellules finit par constituer 4 axes longitudinaux, 2 axes latéro-ventraux et 2 axes latéro-dorsaux (fig. 2 A et $\mathrm{B}$, fin du $1^{\text {er }}$ stade).

Ces axes sont séparés les uns des autres par des bandes longitudinales cytoplasmiques où l'on reconnâit 1 ou 2 files de noyaux (fig. 2 B). La structure syncitiale de ces bandes ainsi que leur situation (il existe 2 bandes latérales, 1 bande dorsale et 1 bande ventrale) montrent qu'elles correspondent aux futures cordes ectodermiques de la Filaire adulte.

Toutes les cellules filles de R1 se trouvent ainsi situées entre ces cordes, à l'emplacement des futurs champs musculaires, dont elles sont les initiales. Au stade I, quand la larve est immobile, ces cellules ont un cytoplasme encore indifférencié, non contractile; la différenciation est réalisée chez la larve infestante (cf. Feng., 1936; Schacher, 1962).

2) Autres espèces observées.

- $W$. bancrofti, au début du développement les phénomènes sont identiques ; R1 engendre 4 cellules filles disposées dans les axes latéro-ventraux et latéro-dorsaux qui n'ont aucun lien avec le tube digestif (fig. $2 \mathrm{C}, \mathrm{D}, \mathrm{E}, 67^{\circ} \mathrm{h}$ ). Par la suite, le manque de netteté des limites cellulaires n'a pas permis de suivre la formation des axes cellulaires latéro-ventraux et latéro-dorsaux ; toutefois, chez les larves en vue latérale, on observe généralement au niveau de la jonction intestin-rectum quelques grosses cellules à noyaux volumineux qui semblent provenir de R1; ces cellules ne sont pas en contact avec la cuticule mais séparées d'elle par une mince couche cytoplasmique appartenant au revêtement sous-cuticulaire (fig. $2 \mathrm{~F}, 108^{\circ} \mathrm{h}$ ).

- F. candezei, cette Filaire est trop exiguë pour étudier les premières transformations de R1; mais plus tard, on retrouve à la hauteur de la jonction intestin-rectum de grosses cellules équivalentes de celles de $W$. bancrofti.

Ces quelques observations nous autorisent à penser que, quelle que soit l'espèce filarienne étudiée, les champs musculaires sont mis en place par la descendance de R1. Ces faits ont le mérite d'être en accord d'une part avec le plan d'organisation du fourreau épithélio-musculaire admis pour les autres Nématodes (Chitwood et Chitwood, 1950) et d'autre part avec les données embryologiques de Martini (1908, in Grassé) ; pour cet auteur, en effet, la musculature est mise en place tardivement à partir d'initiales refoulées dans l'épaisseur de l'embryon qui se multiplient et s'organisent en bandes mésoblastiques; celles-ci viennent s'appliquer sur la face interne du revêtement ectodermique.

\section{Conclusion.}

L'étude des premières heures du développement des microfilaires chez l'hôte intermédiaire permet de préciser la fonction de certaines cellules particulières et l'origine de quelques organes: les cellules R2 à R4 servent à l'élaboration du rectum ; la cellule R1 est à l'origine de la musculature de la Filaire adulte ; l'intestin se forme 


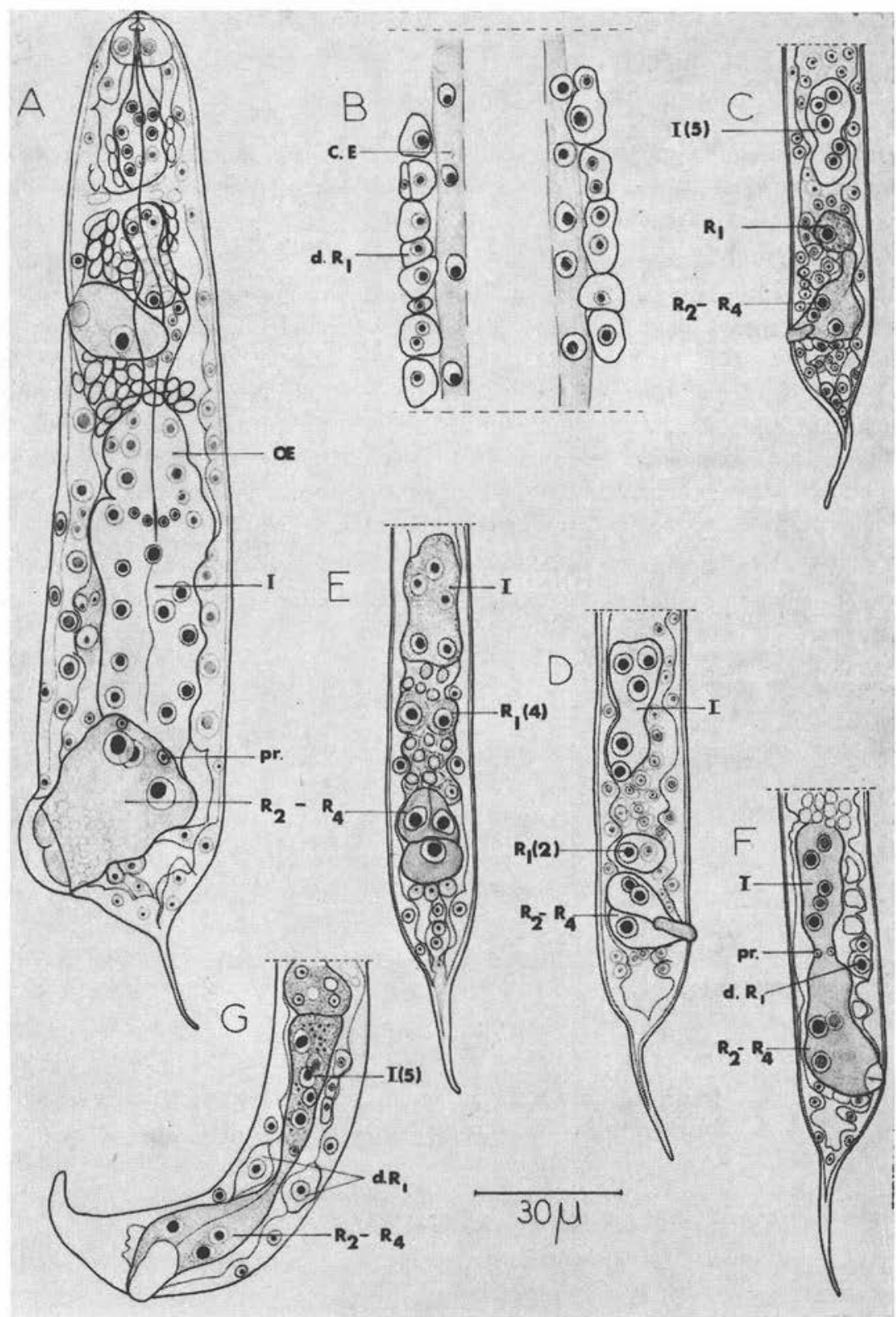

Fig. 2. - A : S. labiatopapillosa, $95^{\mathrm{e}} \mathrm{h}$, vue latérale, en coupe optique épaisse (ventralement, les 2 couches du revêtement de la larve, ectodermique et mésodermique normalement décalées, sont représentées et paraissent se superposer); 2 petites cellules prérectales sont visibles. - B : idem, vue superficielle des cordes syncitiales ectodermiques et des files de grandes cellules issues de R1 (axes latéroventral et latérodorsal). - C à F: $W$. bancrofti; $\mathrm{C}: 67^{\circ} \mathrm{h} ; \mathrm{le}$ bourgeon intestinal est formé de 5 cellules, R1 n'est pas divisée, le rectum est formé par R2 à R $4-\mathrm{D}$ : idem, la cellule $\mathrm{R} 1$ est divisée une fois. - E: idem, vue dorsale; la cellule R1 a donné 4 cellules filles; F : idem, 4 jours $1 / 2$; à la jonction entre l'intestin et le rectum se trouvent les 2 petites cellules prérectales; la descendance de R1 n'est représentée que sur la face ventrale et paraît artificiellement être dans l'axe ventral médian. - G : F . candezei, $5^{e}$ jour ; ici encore pour faciliter le dessin, les descendants de R1 paraissent être situés dans les plans médians; une seule cellule du prérectum est représentée (éch. $30 \mu$ ) 
à partir de 5 initiales qui ne sont pas visibles chez la microfilaire ; l'œsophage se construit autour d'un axe cuticulaire qui s'étend de l'extrémité apicale jusqu'au voisinage du noyau intestinal le plus antérieur.

En dépit de sa silhouette allongée et de sa mobilité, la microfilaire apparaît ainsi comme un authentique embryon. Il présente 2 invaginations ectodermiques aux extrémités du corps; dans la région postérieure, 3 cellules ectodermiques sont invaginées (R2, R3, R4) et conservent la capacité de sécréter de la cuticule (bouchon anal); dans la région antérieure, l'invagination n'est visible que par la présence de l'axe chitinoïde œsophagien, mis parfois en évidence seulement par des colorations histochimiques (Laurence et Simpson, 1968). L'endoderme de cet embryon est constitué de 5 cellules (cellules intestinales). A la limite entre l'endoderme et l'ectoderme postérieur invaginé, se trouve le mésoderme réduit à 1 cellule (cellule R1).

La forme très allongée de la microfilaire, de même que l'existence d'un ectoderme contractile, apparaissent comme une adaptation secondaire au milieu liquide où circule cet embryon.

\section{REMERCIEMENTS}

- Nous exprimons toute notre gratitude à MM. Brengues et Gidel qui ont effectué l'infestation du veau. filariens.

- Nous remercions vivement $M$. Prod'hon qui nous fait parvenir les Caméléons

- Nous tenons à exprimer tout particulièrement notre reconnaissance à $\mathbf{M}^{\mathrm{me}}$ Escaig (Laboratoire des Etres Organisés, boulevard Raspail, Paris) qui a effectué les coupes et les colorations pour l'étude électronique.

\section{Bibliographie}

BaIN (O.), 1969 a. - Morphologie des stades larvaires d'Onchocerca volvulus chez Simulium damnosum et redescription de la microfilaire. Ann. Parasit. Hum. et Comp., 44, p. 69-82.

Barn (O.), $1969 b$. - Etude morphologique du développement larvaire de Foleyella furcata chez A. stephensi. Ann. Parasit. Hum. et Comp., 44, p. 165-172.

BaIn (O.) (sous presse). - Développement larvaire de Setaria labiatopapillosa chez Aedes aegypti. Ann. Parasit. Hum. et Comp.

Chitwood (B. G.) et ChItwood (M. B.), 1950. - An introduction to Nematology. Section I. Anatomy. Baltimore.

FENG (L. C.), 1936. - The development of Microfilaria malayi in A. hyrcanus var. sinensis. Chinese. Med. J., suppl. I, p. 345-367.

IYENGAR (R.), 1956. - Développement de Wuchereria bancrofti (Cobbold) et de Wuchereria malayi (Brug.). Ann. Parasit. Hum. et Comp., 31, p. 99-138 et 266-287.

Kobayashi (Н.), 1940. - On the development of Microfilaria bancrofti in the body of the mosquito (Culex fatigans). Acta. Jap. Med. Trop., 2, p. 63-88. 
Laurence (B. R.) et Simpson (M. G.), 1968. - Cephalic and pharyngeal structures in microfilariae revealed by Staining. J. Helminth., 42, p. 309-330.

Martini (E.) in Grassé (P.-P.), 1965. - Traité de Zoologie. Némathelminthes, 4 (2), 731 p., Edit. Masson et $\mathrm{C}^{\mathrm{ie}}$, Paris.

Nigon (V.) in Grassé (P.-P.), 1965. - Traité de Zoologie. Némathelminthes, 4 (2), 731 p., Edit. Masson et $\mathrm{C}^{\mathrm{ie}}$, Paris.

Rodenwaldt (E.), 1908, - Die Verteihung der Microfilaria im Körper und die Ursachen des Turnus bei Mikrofilarien nocturna und diurna Studien zur Morphologie der Mikrofilarien. Arch. f. Schiffs-u. Tropenhyg., 12, 18-30.

SCHACHER (J.), 1962. - Morphology of the microfilaria of Brugia pahangi and of the larval stages in the mosquito. J. Parasit., 48, p. 679-692.

TAYLOR (A. E. R.), 1960. - Studies on the microfilariae of Loa loa, Wuchereria bancrofti, Brugia malayi, Dirofilaria immitis, D. repens and D. aethiops. J. Helminth., 34, p. 13-26. 